\title{
New Strategies for Perioperative Nutrition Control for Patients with Locally Advanced Oral Cancer
}

\section{Shin-ichiro Hiraoka ${ }^{1}$ and Makoto Adachi ${ }^{2 *}$}

${ }^{1}$ First Department of Oral \& Maxillofacial Surgery, Osaka University, Graduated school of Dentistry, Japan

${ }^{2}$ Department of Head and Neck Surgery, The University of Texas MD Anderson Cancer Center, USA

The treatment of oral cancer consists mainly of surgical resection, chemotherapy and radiotherapy. The majority of patients with locally advanced oral cancer undergo surgical resection which, to maintain an optimal surgical margin, often involves facial deformities and loss of oral functions such as drinking, mastication, swallowing, and speaking. On the other hand, patients treated with chemotherapy and radiotherapy typically have severe stomatitis and pharyngitis, which make eating difficult. Furthermore, a majority of oral cancer patients are nutritionally compromised at the time of diagnosis because of dysphagia or odynophagia from the primary tumor. Nutritional compromise is associated with increased morbidity and mortality in oral cancer patients. Nutritionally compromised patients have greater susceptibility to postoperative complications such as infection and delay of wound healing, poorer response to treatment, and higher likelihood of recurrence after treatment.

Despite the recognized importance of nutrition, the optimal approach to maintaining nutrition for the oral cancer patient is unclear. There are controversies surrounding route, timing, nutrients and amount of feeding. Although intravenous hyperalimentation has often been used to manage perioperative nutrition in patients who have a disability limiting oral intake, recent guidelines recommend tubefeeding via a nasogastric tube (NG tube) or Percutaneous Endoscopic Gastrostomy (PEG) [1].

Enteral feeding, or tubefeeding, is now recommended for several reasons. Fasting (including Total Parenteral Nutrition [TPN]) for as few as 3 days causes atrophy of the intestinal epithelium and the Peyer's patch, leading to Bacterial Translocation (BT). Because the intestine takes in $70 \%$ of nutrients, including glutamine, from the lumen, enteral feeding protects the intestinal structure and prevents BT. Nourishment dosage is more difficult to control with TPN, and surplus nourishment dosage can cause hyperglycemia, lipid abnormalities, liver damage and other serious complications. Compared to TPN, enteral nourishment is much closer physiologically to normal food intake and is rarely complicated by hyperglycemia, lipid abnormalities or liver damage. TPN also is associated with catheter infection. Finally, being more direct, enteral nourishment requires a lower dosage, approximately one-third that of TPN, and thus is much less costly.

We now know that some key nutrients can modulate the immune system and metabolic pathways, an approach referred to as nutritional pharmacology. When the nutrients contribute specifically to modulation of pathways clearly associated with inflammation or metabolism, it is called immunonutrition [2]. Although many nutrients modify these pathways, most clinical interest has focused on arginine, glutamine, fatty acids (omega-3 polyunsaturated fatty acids, or $\omega-3$ PUFA), ribonucleotides and certain trace elements. These nutrients not only improve nutritional status, they also promote wound healing $[3,4]$ and improve prognosis [5]

In recent years, eicosapentaenoic acid (EPA), a $\omega-3$ PUFA extracted from fish oil, has been used as a nutritional supplement in cancer patients [6]. Resolving, a metabolite of EPA, strongly inhibits inflammatory cytokines [7]. Fearon et al. [8] reported the findings of a prospective study in which patients with incurable pancreatic cancer received a nutritional regimen that included EPA for 4-8 weeks. The investigators concluded that this regimen slowed loss of body weight and improved quality of life.

Tubefeeding is frequently associated with diarrhea, however, and diarrhea causes under nutrition as well as electrolyte imbalance, which increase risk for postoperative complications. General treatment of this diarrhea has been adjustment of the tubefeeding to a slower inflow speed. A newer strategy is administration of probiotics and prebiotics. Probiotics are useful microbes that promote growth of beneficial microorganisms in the host's gastrointestinal tract. When taken orally, probiotic microbes act on the digestive flora to modulate the immune system and prevent illness while doing no harm to the digestive tract [9]. Prebiotics, on the other hand, are oligosaccharides that help probiotics work; because they are not broken down or absorbed in the upper gastrointestinal tract, they nourish the enteral probiotics, helping to restore a healthy enteral environment and improve health and well-being [10].

Despite the presence of high levels of bacteria in the oral cavity, which promote infection and compromise wound healing after surgery, there are no published reports of nutrition trials in patients undergoing treatment for oral cancer. Therefore, we are carrying out a trial of Prosure ${ }^{\varpi}$ (Abbott Laboratories, Abbott Park, Illinois, USA), a nutritional formulation rich in EPA, in patients with oral cancer. The patients also receive a prebiotic known as $\mathrm{YH}$-Flore $^{\bullet}$ (Meiji, Tokyo, Japan) to reduce diarrhea during the perioperative period. The combination of Prosure ${ }^{\oplus}$ and $\mathrm{YH}$-Flore $^{\oplus}$ may prevent or lessen complications such as delay of surgery wound healing and infection of the wound and may allow continuation of tubefeeding for postoperative nutrition without diarrhea and other complications.

Perioperative nutrition in oral cancer patients is controversial. More prospective trials will improve nutritional outcome and shortand long-term consequences for oral cancer patients who must undergo surgery. We also hope to provide new evidence to guide perioperative nutrition for oral cancer patients in the future.

*Corresponding author: Dr. Makoto Adachi, DDS, PhD, Department of Head and Neck Surgery, The University of Texas MD Anderson Cancer Center, 1515 Holcombe Boulevard, Unit 0123, Houston, Texas 77030, USA, Tel: +1-713-5634766; Fax: +1-713-754-2234; E-mail: madachi_dds_phd@yahoo.co.jp

Received June 29, 2012; Accepted July 02, 2012; Published July 04, 2012

Citation: Hiraoka SI, Adachi M (2012) New Strategies for Perioperative Nutrition Control for Patients With Locally Advanced Oral Cancer. Dentistry 2:e109. doi:10.4172/2161-1122.1000e109

Copyright: (c) 2012 Hiraoka SI, et al. This is an open-access article distributed under the terms of the Creative Commons Attribution License, which permits unrestricted use, distribution, and reproduction in any medium, provided the original author and source are credited. 
Citation: Hiraoka SI, Adachi M (2012) New Strategies for Perioperative Nutrition Control for Patients With Locally Advanced Oral Cancer. Dentistry 2:e109. doi:10.4172/2161-1122.1000e109

Page 2 of 2

\section{Acknowledgements}

We thank Akira Ito, Shunsuke Nagatani, Katsumi Azenishi (First Department of Oral \& Maxillofacial Surgery, Osaka University, Graduated school of Dentistry) for discussion and assistance. We particularly thank the Dentistry editorial team. Makoto Adachi was supported by the Uehara Memorial Foundation Postdoctoral Fellowship and the Japan Society for the Promotion of Science Postdoctoral Fellowship for Research Abroad.

\section{Conflict of Interest Statement}

The authors declare that they have no conflict of interest.

\section{References}

1. Pittiruti M, Hamilton H, Biffi R, MacFie J, Pertkiewicz M (2009) ESPEN Guidelines on Parenteral Nutrition: central venous catheters (access, care, diagnosis and therapy of complications). Clin Nutr 28: 365-377.

2. Zhang Y, Gu Y, Guo T, Li Y, Cai H (2012) Perioperative immunonutrition for gastrointestinal cancer: a systematic review of randomized controlled trials. Surg Oncol 21: e87-e95.

3. Stechmiller JK, Childress B, Cowan L (2005) Arginine supplementation and wound healing. Nutr Clin Pract 20: 52-61.

4. Okamoto Y, Okano K, Izuishi K, Usuki H, Wakabayashi H, et al. (2009) Attenuation of the systemic inflammatory response and infectious complications after gastrectomy with preoperative oral arginine and omega-3 fatty acids supplemented immunonutrition. World J Surg 33: 1815-1821.

5. Rosa F, Bossola M, Pacelli F, Alfieri S, Doglietto GB (2011) Malnutrition and postoperative complications in abdominal surgery. Ann Surg 254: 666

6. Fearon KC, Barber MD, Moses AG, Ahmedzai SH, Taylor GS, et al. (2006) Double-blind, placebo-controlled, randomized study of eicosapentaenoic acid diester in patients with cancer cachexia. J Clin Oncol 24: 3401-3407.

7. Chapkin RS, Kim W, Lupton JR, McMurray DN (2009) Dietary docosahexaenoic and eicosapentaenoic acid: emerging mediators of inflammation. Prostaglandins Leukot Essent Fatty Acids 81: 187-191.

8. Fearon KC, Von Meyenfeldt MF, Moses AG, Van Geenen R, Roy A, et al. (2003) Effect of a protein and energy dense N-3 fatty acid enriched oral supplement on loss of weight and lean tissue in cancer cachexia: a randomised double blind trial. Gut 52: 1479-1486.

9. Drisko JA, Giles CK, Bischoff BJ (2003) Probiotics in health maintenance and disease prevention. Altern Med Rev 8: 143-155.

10. Bouhnik Y, Raskine L, Simoneau G, Vicaut E, Neut C, et al. (2004) The capacity of nondigestible carbohydrates to stimulate fecal bifidobacteria in healthy humans: a double-blind, randomized, placebo-controlled, parallel-group, doseresponse relation study. Am J Clin Nutr 80: 1658-1664. 